\title{
The application of movable water saturation from the calculation of neural network method
}

\author{
Wei Xiong ${ }^{1,2}$,Jia-Feng $\mathrm{Wu}^{1}$,Shu-Sheng Gao ${ }^{1,2}$, Li-You Ye ${ }^{1,2}$ \\ and Fei-Fei Gou ${ }^{1}$ \\ ${ }^{1}$ Institute of Porous Flow and Fluid Mechanics, Chinese Academy of Sciences, \\ Langfang, Hebei, 065007, China; \\ 2.Research Institute of Petroleum Exploration and Development-Langfang, \\ Hebei, 065007, China
}

\begin{abstract}
Movable water saturation can accurately evaluate the formation of gas and water layer. It is an important parameter to guide the development of tight gas reservoirs. Engineering often used single factor of porosity to estimate saturation of the movable water. And in the formation of strong heterogeneity conditions, precision linear regression method will be affected, BP neural network rule can be a good fit multivariate highly nonlinear problems. Combining multiple factors logs to establish BP neural network model to calculate the formation of the movable water saturation, and applied to other wells area to verify the distribution of gas and water layer. The results show: at formation heterogeneity strong case, BP neural network computing movable water saturation with sufficient accuracy, and can accurately predict the characteristics of water production in gas well.
\end{abstract}

Keyword: movable water saturation; nonlinear; neural network; predict the characteristics of water production

\section{Introduction}

Developing unconventional oil and gas resources will be the key point for the output of conventional oil and gas is declining. Reserves of unconventional oil and gas outclasses conventional oil and gas's reserves, so it has much value for developing [8]. Tight sandstone gas reservoir belong to unconventional gas resources, technology for developing this reservoirs is also the ripest. But the most important technology is that if we can evaluating the property of fluid in the stratum. Saturation of mobile fluid is a critical parameter for predicting if the tight reservoir's stratum yield water. Bond water is necessary for calculating saturation of mobile fluid. And there exists high nonlinear relationship between bond water and the other parameters of stratum, such as pore structure, formation water salinity and temperature etc. So it is very tough for calculating bond water with a very high precision. Generally the method to calculate the bond water can be acquired by porosity and bond water value obtained by 
nuclear magnetic resonance in the lab, then saturation of mobile fluid can be got, but nuclear magnetic resonance experiment need a lot of core, which is not convenience for application. BP neural network method can match the high nonlinear relationship and have strong error-tolerance and high precision. In the paper we get saturation of mobile fluid by BP neural network method with using six kinds of log curves and interpret gas bearing layer and water bearing layer.

\section{Calculation Theory}

\section{BP neural network}

Artificial neural network is established by basing on the principle of biological neural network working. Artificial neuron is the basic unit of artificial neural network, which is almost equivalent to a multiple-input single-output nonlinear threshold devices (Figure 1). Each neuron receives information from each neurons which belongs to adjacent layers and also send messages to those neurons. Artificial neural network processes information through the interaction of neurons[1]. In this paper, BP neural network will be used to solve the problem presented above.
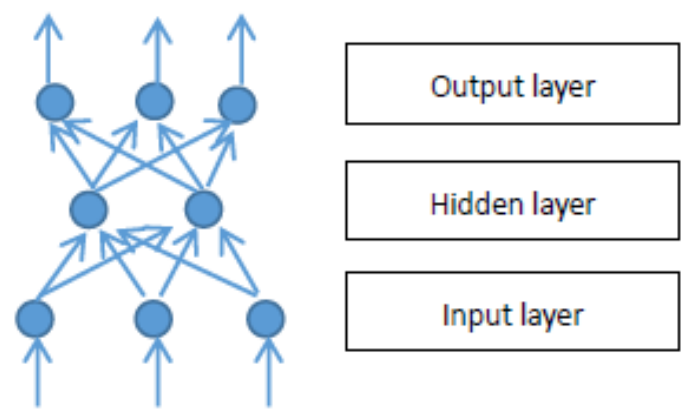

Fig.1 Typical neural network structure

As shown in Figure 1, BP neural network can be divided into three parts of the input layer, hidden layer and output layer, input layer and output layer are connected by a hidden layer, and we can choose one or more layers as the hidden layer. The neurons are not connected with each other in the same layer, but neurons are connected in adjacent layers. Input layer receives information, the number of neurons depends on the input vector dimension. Output layer yield processed information, the number of neurons of output layer depends on the desired output vector dimension[1]. Usually S (sigmoid) function can be the transfer function of BP network:

$$
f(x)=\left(1+e^{-x}\right)^{-1}
$$

Wheref(x)is a sigmoid monotone threshold function.f $(\mathrm{x}) \in[0,1] .[1,2,4]$ 
$S$ function ensure that non-linear function of neurons, network consists of many non-linear neurons, which has a highly non-linear mapping. Enter information spread in the network with the following ways:

$$
\begin{aligned}
X_{i} & =\sum_{j} W_{j i} Y_{1 j}-\theta_{i} \\
Y_{2 j} & =f\left(X_{i}\right)
\end{aligned}
$$

Where $\mathrm{X}_{\mathrm{i}}$ is weighted inputs received by input layer neuron $\mathrm{I} ; \mathrm{W}_{\mathrm{ji}}$ is connection weights which is inputted into input layer neuron $i$ by hidden layer neuron $\mathrm{j} ; \theta_{\mathrm{i}}$ is the threshold of input layer neuron $I ; Y_{1 j}$ is the output value of hidden layer neuron $j ; Y_{2 j}$ is the output value of the output layer. [2,3,4]

The networks with three layerscan have the following mathematical expression:

$$
D_{i}=f\left[\sum_{j} W_{j i}^{(1)} f\left(\sum_{k} W_{k j}^{(2)} X_{k}-\theta_{2 j}\right)-\theta_{1 j}\right]
$$

Where $X_{k}$ is input variables; $D_{i}$ is output; $W_{j i}^{(1)}$ is connection weights between output layer neuronsand hidden layer neurons; $\mathrm{W}_{\mathrm{kj}}^{(2)}$ is connection weights between input layer neurons and hidden layer neurons; $\theta_{1 \mathrm{j}}$ is the threshold of output layer neuron; $\theta_{2 j}$ is the threshold of hidden layer neuron. $[2,3,4]$

\section{Method of Well Logging Interpretation on Saturation of Mobile Fluid Based on Neural Network}

\section{Theory of movable water saturation log interpretation}

The aqueous phase mainly exists in the micro and small pores, the gas phase is almost in the macro-pore, which leads to form gas-water interaction sealed state. During the developing process, the pressure of reservoir goes down, so the gas expansion can make bound water yield, then the bound water become movable water. And movable water saturation can directly reflect the characteristics of gas wells producing water.

Movable water saturation and irreducible water saturation have a following relation:

$$
S_{w m}=S_{w}-S_{w r}
$$

Where $S_{w m}$ is movable water saturation, $S_{w}$ is original water saturation, $\mathrm{S}_{\mathrm{wr}}$ is irreducible water saturation. Original water saturation is obtained from well logs, we can get the logging curves of movable water saturation as long as obtaining the model of calculating irreducible water saturation with enough accuracy, then we can interpret gas bearing layer and water bearing layer. 


\section{Multi-Parameter Model of Irreducible Water Saturation}

\section{Selecting parameters for logging}

There exist many factors which can affect the irreducible water saturation, such as types of sandstone, lithology, clay content, median particle size, porosity, pore structure, formation water salinity, temperature, formation pressure. Porosity, pore structure, clay content and formation water salinity are the main factors.

Acoustic time $(\mathrm{AC})$ and neutron porosity $(\mathrm{CNL})$ can reflect porosity and pore structure. Natural potential (SP) and gamma ray (GR) can present the content of clay. Deep lateral resistivity log (LLD) and shallow lateral resistivity logging (LLS) can describe formation water salinity. Therefore we choose the six logging curves as the basis for calculation.

\section{Logging data preprocessing}

We select the 82 cores $(3300 \mathrm{~m} \sim 3500 \mathrm{~m})$ which come from the four wells: X1, $\mathrm{X} 2, \mathrm{X} 3, \mathrm{X} 4$, all the wells belong to the development block of Su 75. The we carry out true depth determination of core, which make the depth of logging be the same as the true depth of core. 
Table1-1 Core location after the original logging data(partial data)

\begin{tabular}{|c|c|c|c|c|c|c|c|c|c|}
\hline $\begin{array}{c}\text { Well } \\
\text { number }\end{array}$ & $\begin{array}{c}\text { Core } \\
\text { number }\end{array}$ & $\begin{array}{c}\text { Location } \\
\text { depth(m) }\end{array}$ & AC & GR & SP & LLD & LLS & CNL & $\begin{array}{c}\text { Irreducible } \\
\text { water } \\
\text { saturation } \\
\text { of } \\
\text { NMR(\%) }\end{array}$ \\
\hline $\mathrm{X} 1$ & $1-26-33$ & 3332.84 & 228.11 & 86.91 & 89.55 & 21.96 & 18.44 & 6.47 & 67.90 \\
\hline $\mathrm{X} 1$ & $1-23-33$ & 3332.18 & 224.90 & 82.33 & 87.83 & 27.37 & 22.46 & 6.56 & 64.29 \\
\hline $\mathrm{X} 1$ & $1-22-33$ & 3331.85 & 224.41 & 61.57 & 87.09 & 28.51 & 23.60 & 6.00 & 68.51 \\
\hline $\mathrm{X} 1$ & $1-20-33$ & 3331.52 & 222.05 & 49.79 & 86.67 & 32.31 & 34.43 & 5.34 & 53.40 \\
\hline $\mathrm{X} 1$ & $1-18-33$ & 3330.86 & 230.57 & 63.74 & 87.06 & 25.43 & 21.16 & 5.76 & 58.84 \\
\hline $\mathrm{X} 1$ & $1-19-33$ & 3331.19 & 227.60 & 61.57 & 86.69 & 24.43 & 21.06 & 5.74 & 55.01 \\
\hline $\mathrm{X} 2$ & $1-36-37$ & 3439.59 & 218.70 & 58.39 & 39.42 & 53.63 & 53.28 & 8.02 & 57.78 \\
\hline $\mathrm{X} 2$ & $1-35-37$ & 3439.32 & 224.73 & 49.69 & 36.98 & 40.54 & 46.31 & 10.09 & 70.25 \\
\hline $\mathrm{X} 2$ & $1-34-37$ & 3439.05 & 232.82 & 56.65 & 34.27 & 43.95 & 46.49 & 12.65 & 56.43 \\
\hline $\mathrm{X} 2$ & $1-7-37$ & 3433.38 & 226.04 & 46.64 & 20.19 & 55.48 & 57.35 & 11.08 & 54.08 \\
\hline $\mathrm{X} 2$ & $2-33-35$ & 3481.95 & 236.61 & 48.07 & 22.46 & 24.86 & 27.49 & 8.80 & 62.11 \\
\hline $\mathrm{X} 4$ & $2-16 / 42$ & 3489.88 & 217.54 & 48.27 & 201.63 & 97.15 & 109.13 & 8.35 & 49.63 \\
\hline
\end{tabular}

\section{Developing model}

Network model consists of an input layer, a hidden layer and an output layer. The network structure of this model has six nodes, corresponding to six sets of $\log$ data which are the input variables:AC, GR, SP, LLD, LLS, CNL, hidden layer has 20 nodes, the output layer has 1 node, the number of training samples are 58. Output data can be described by input data and activation function:

$$
\mathrm{y}=\operatorname{NET}(\mathrm{AC}, \mathrm{GR}, \mathrm{SP}, \mathrm{LLD}, \mathrm{LLS}, \mathrm{CNL})=\sum_{\mathrm{i}=1}^{\mathrm{m}} \mathrm{W}_{2} \operatorname{tansig}\left(\mathrm{W}_{1} \mathrm{X}+\theta_{\mathrm{i}}^{1}\right)+\theta_{\mathrm{i}}^{2}(6)
$$

Where $\mathrm{X}=(\mathrm{AC}, \mathrm{GR}, \mathrm{SP}, \mathrm{LLD}, \mathrm{LLS}, \mathrm{CNL})-1, \mathrm{~W}_{1}$ is the weights between input layer and hidden layer, $\mathrm{W}_{2}$ is the weights between output layer and hidden layer, $\mathrm{m}$ is the number of neurons in neural networks, $\theta_{\mathrm{i}}^{1}$ is $\mathrm{i}$-th threshold of the 
first layer, $\theta_{\mathrm{i}}^{2}$ is $\mathrm{i}$-th threshold of the second layer,tansig is transmission function of neural network, NET is the corresponding network.

\section{Network training}

Training results acquired by Matlab: $W_{1}$ :

$\left[\begin{array}{cccccc}-1.25837 & -1.25684 & -2.40889 & 0.209734 & -0.637 & -1.36232 \\ 1.204427 & -0.88894 & 1.216916 & 1.040377 & -0.12967 & 0.650134 \\ -0.98854 & -1.10979 & 0.09506 & -0.93424 & -0.93946 & -1.16008 \\ -1.66947 & 0.828693 & 1.882841 & 1.082503 & 1.874205 & -1.28973 \\ -0.87032 & -0.32387 & 1.786681 & 1.263583 & 0.150788 & 0.728079 \\ -2.11469 & -0.20189 & 0.07524 & -0.24821 & -1.37758 & 0.74888 \\ -0.00783 & -0.49201 & -1.84748 & -1.57777 & -2.35733 & -0.10157 \\ 0.928519 & 0.725971 & -0.47526 & 1.113633 & -1.26581 & 1.581398 \\ -1.27431 & 0.565375 & 1.651892 & -1.13434 & -1.93257 & 0.665534 \\ -0.3844 & -1.80209 & 0.363402 & -0.90615 & 0.242807 & 0.572485 \\ -0.28249 & 2.212572 & -2.46373 & -1.67443 & 0.720689 & 0.269192 \\ 1.27874 & -0.08313 & -2.06721 & 1.342689 & 0.41593 & 0.980373 \\ -1.06032 & 0.623135 & -0.41349 & -1.56844 & -1.1886 & -0.22066 \\ 1.357537 & -0.3768 & -0.67567 & -0.82214 & -1.24922 & -0.6176 \\ 0.238302 & -0.01403 & -0.21627 & -1.04108 & 0.548896 & -1.45115 \\ 1.99684 & -0.10662 & -0.66798 & -0.02033 & 1.281158 & -0.58666 \\ 1.571633 & -0.2806 & -0.57982 & -1.80381 & -0.09096 & -0.0272 \\ 0.937893 & 1.382038 & -0.8316 & 1.520472 & -0.91426 & 0.344717 \\ -0.15713 & -0.92401 & 0.741115 & -1.53462 & 0.70974 & 0.608845 \\ -0.02559 & -0.02197 & 0.886307 & -1.45657 & -0.79327 & 0.024198\end{array}\right]$

$W_{2}$ :

$(-1.519616035,-0.438770173,0.485543901,-0.131901444$

$-0.772990904,0.225059083,-0.926997039,-1.262936132$,

$-0.338087644,0.255149593,1.597526673,0.441554379,0.120762173$,

$-0.174374669,0.731562593,-1.484760468,-0.283678616,-1.823292892$,

$-0.171543163,0.595294806)^{-1}$

The expression equation of calculation irreducible water saturation:

$$
y=W_{2} f\left(W_{1} x-\theta_{1}\right)-\theta_{2}(7)
$$

Where $\theta_{1}:(1.817978,-2.10477,1.817729,1.064861,0.381822,1.857974$, $-0.19345,-0.78383,-0.10717,0.509094,-0.64286,0.068176,-0.29869$, $0.837876,1.461297,1.631246,1.460624,1.874247,2.497295$,

\section{$-2.76768) ; \theta_{2}: 0.728728$}

Excitation function $\mathrm{f}(\mathrm{x})$ has the following expression:

$$
f(x)=\frac{2}{1+e^{-2 x}}-1
$$

\section{Network training}

Some of the data are used to train the model for establish of BP neural network and another data are used to test or validate the model. Corresponding, there are 
3 errors in the model: train error, test error and validate error. We usually use MSE(Mean Square Error) and the correlation coefficient to measure the network performance. The MSE is smaller and the correlation coefficient close 1, the network reliability is better. [12]Irreducible water saturation is predicted $\mathrm{y}_{\text {out,i }}(\mathrm{X})$ by the BP network, and the experimental data is defined to $\mathrm{y}_{\mathrm{r}, \mathrm{i}}(\mathrm{X})$. The MSE of the BP network is that:

$$
\operatorname{MSE}=\frac{1}{\mathrm{n}} \sum_{1}^{\mathrm{n}}\left[\mathrm{y}_{\text {out }, \mathrm{i}}(\mathrm{X})-\mathrm{y}_{\mathrm{r}, \mathrm{i}}(\mathrm{X})\right]^{2}
$$

Where $\mathrm{X}=(\mathrm{AC}, \mathrm{GR}, \mathrm{SP}, \mathrm{LLD}, \mathrm{LLS}, \mathrm{CNL})-1$, $\mathrm{i}$ is experimental point count, $\mathrm{n}$ is the total number of experimental point.

During BP Network training process, the blue line, the red line and the green line respectively represents the training data groups, the test data groups and the validation data groups. The iteration numbers and the MSE shown in Figure 2:

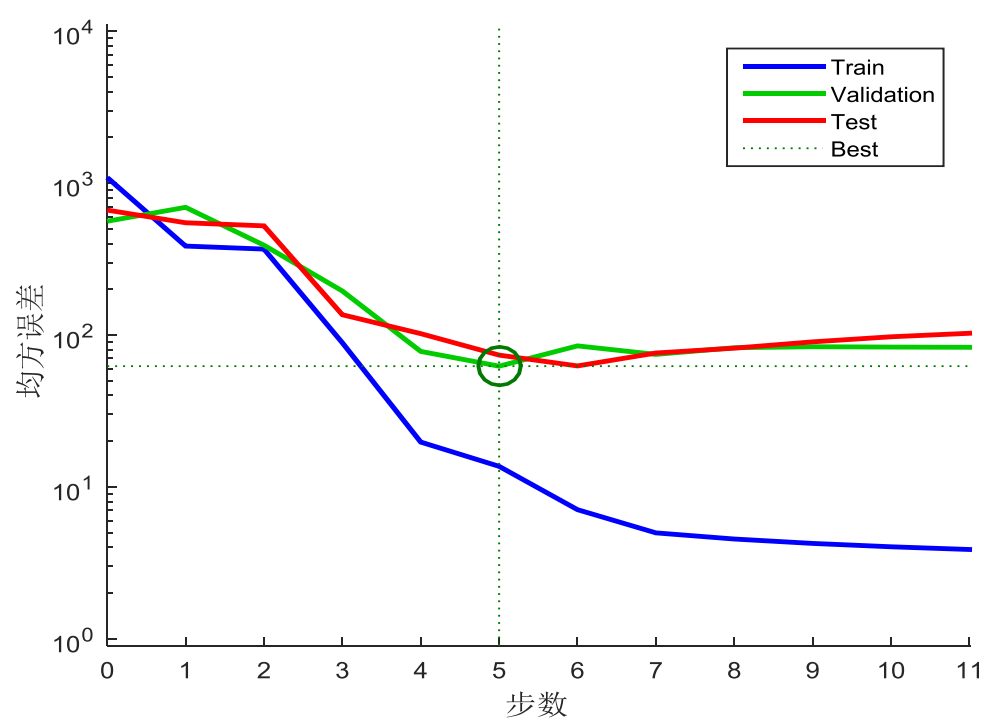

Fig.2 The error of test, train and validation during iteration number changing

The model gets best when the iteration number is 5-step in Figure 2. The train error and the test error shown in Figure 3and Figure 4: 


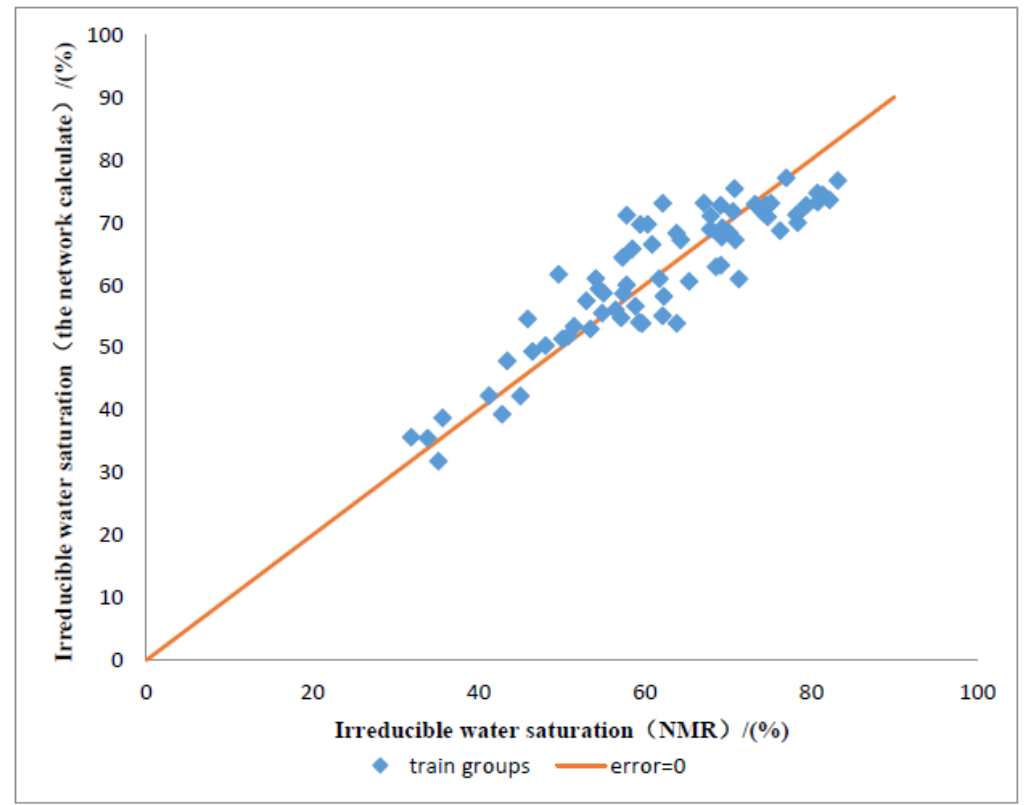

Fig.3 The comparison of the train samples' result of NMR and net

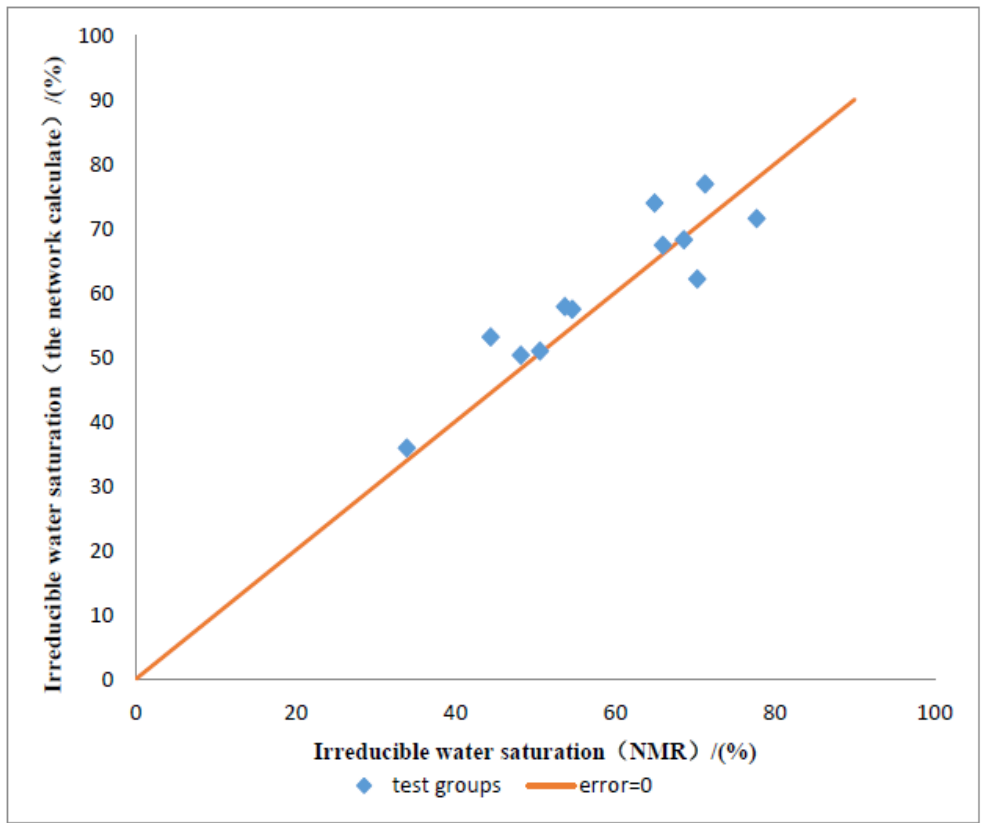

Fig.4 The comparison of the test samples' result of NMR and net 
The average error of train is $9.14 \%$, and test is $6.41 \%$.

\section{The Example of Movable Water Saturation is Used to Log} Interpretation

Irreducible water saturation expression is obtained from BP network according the 6 groups logging data. It defines the movable water saturation distribution of a well X5 as validation based on the movable water saturation definition. That is shown in Figure 5:

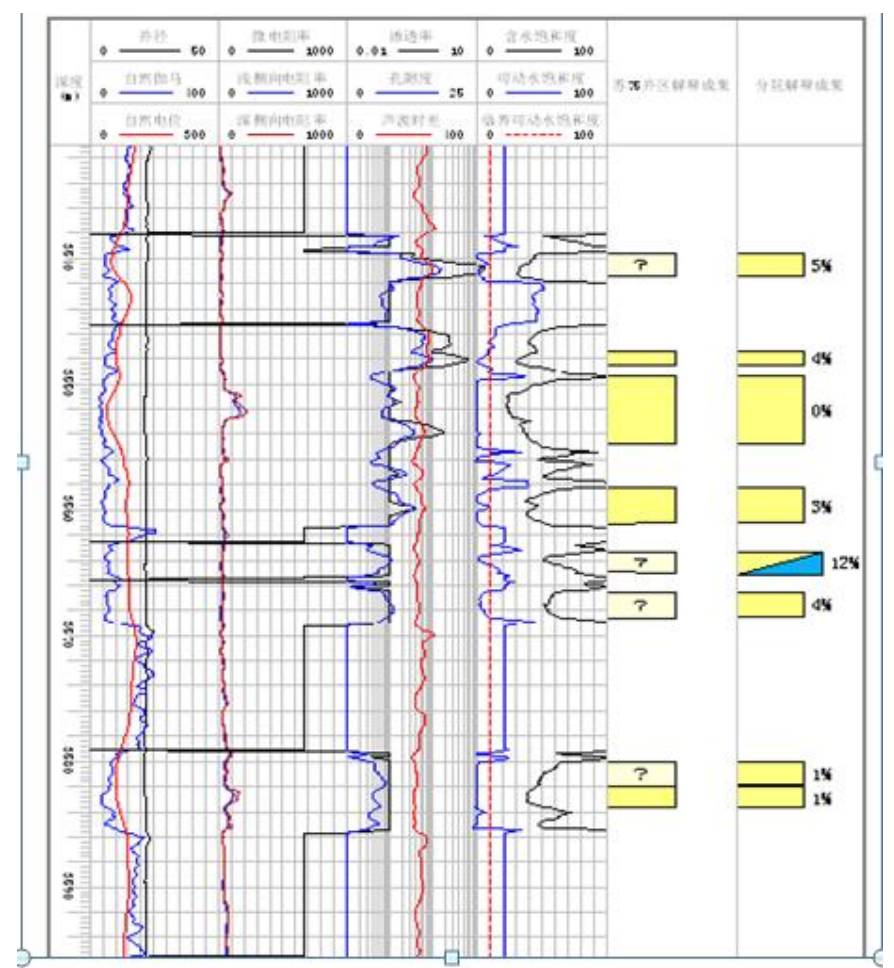

Fig.5 Interpretation of gas and water layer in the well X5

According to the result of the movable reservoir water saturation interpretation, wells producing water features in different layers are predicted as shown in Table 2. The features in different layers can help engineers select the better perforation layer to avoid water production. 
Table 1-2 Interpretation result of gas and water layer in the well X5

\begin{tabular}{cccc}
\hline $\begin{array}{c}\text { Layer } \\
\text { NO. }\end{array}$ & Depth/m & Movable water saturation/\% & Interpretation result \\
\hline 14 & $3539.6 \sim 3541.6$ & 1 & Nowater production \\
16 & $3547.4 \sim 3548.7$ & 4 & No water production \\
17 & $3549.4 \sim 3554.9$ & 0 & No water production \\
19 & $3558.3 \sim 3561.2$ & 2 & No water production \\
20 & $3563.4 \sim 3565.1$ & 12 & A little water production \\
21 & $3566.6 \sim 3568.8$ & 5 & No water production \\
22 & $3580 \sim 3582$ & 1 & No water production \\
23 & $3582 \sim 3583.8$ & 1 & No water production \\
27 & $3602.2 \sim 3604.6$ & 1 & No water production \\
28 & $3624 \sim 3627.2$ & 13 & A little water production \\
\hline
\end{tabular}

The well X5 have gas production $1.8 \times 10^{4} \mathrm{~m} 3 /$ day and have no water production after application of the method. And the cumulative gas production is $575 \mathrm{~m} 3$. There is stable production and achieving the purpose of prevention water production in the well X5. It is shown as Figure 6:

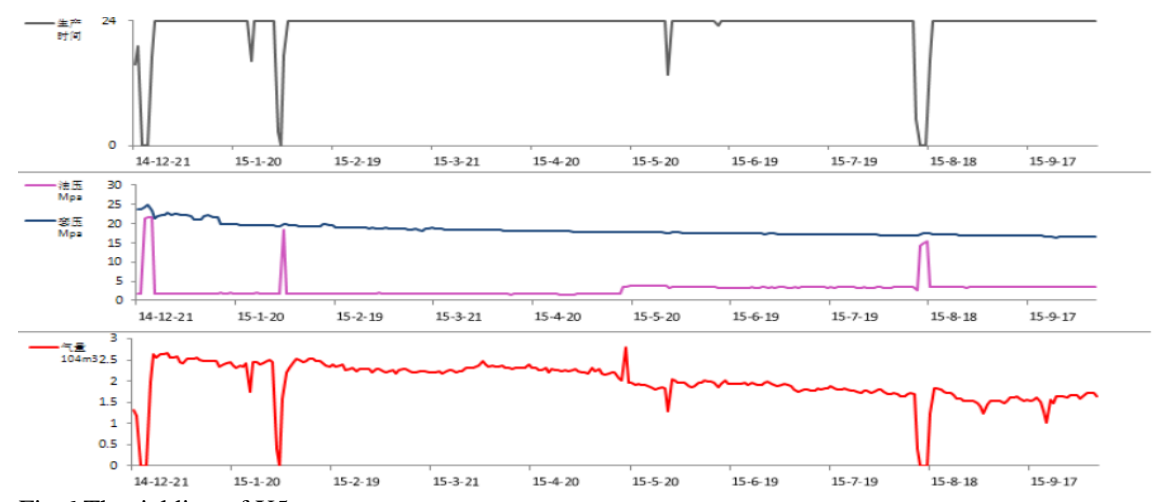

Fig.6 Theyielding of X5

\section{Conclusion}

Irreducible water saturation multi-parameter model is established by BP network from selection the 6 groups log value (AC, GR, SP, LLD, LLS, CNL) as variables to calculation. And the conventional $\log$ interpretation method of 
movable water saturation in tight sandstone reservoir is established basing on this model.

Application of this method to explain the movable water saturation distribution can predict gas wells water production features, guide layers preferably perforation, reducethe risk of gas wells water production.

\section{References}

[1] Gu Jiangrui, Liu Yan. Foreign tight sandstone gas reservoirs Status and trends [J].Foreign Oil Field Engineering, 2009, 25(7):1-5

[2] Ye Liyou, Gao Shusheng, Yang Hongzhi, Xiong Wei, Hu Zhiming, Liu Huaxun, Du Shan. Water production mechanism and development strategy of tight sandstone gas reservoirs [J]. Natural gas industry, 2015, 35 (2):41-46

[3] GaoHua, GaoChuqiao, Hu Xiangyang. Irreducible water saturation Yingge Sea Basin influencing factors [J].Geophysical Prospecting For Petroleum, 2005, 45(2): 158-159

[4] Fu Haijiao, Tang Dazhen, XuHao, et al. Characteristics of tight sandstone reservoir and accumulation process of gas pool [J]. Fault-Block Oil\& Gas Field, 2012, 19 (1); 47-50

[5] Li Xia, Zhao Wenzhi, Zhou Cancan, Wang Tongshan, Li Chaoliu. Dual-porosity saturation model of low-porosity and low-permeability clasticreservoirs $[\mathrm{J}]$. PETROLEUM EXPLORATION AND DEVELOPMENT, 2012, 39(1):82-91

[6] Zhou Kaili, Kang Yaohong. Neural network model and its MATLAB simulation program design [M].Beijing: Tsinghua University press, 2004:1-9, 69-100;

[7] Chen Rong, Wang Feng. Application of BP MATLAB neural network in reservoir physical property forecast [J]. Logging technology, 2009, 33(1): 75-78;

[8] Zhou Wei, Li Rui, Wang Xingwang. Application of BP neural network in the identification of tight sandstone reservoir [J]. Progress in geophysical exploration, 2006, 29(6): 428-432;

[9] Hu Hong, Li Qiang, Xiong Yuqin, Zhang Cheng. The model of oil gas water layer interpretation based on BP artificial neural network [J].Mud logging technology, 2000, 11(4): 13-18;

[10] Fan Huixian, Yang Rui. BP neural network gas water identification research $[\mathrm{J}]$. New technology and new products of China, 2012, (7): 14-15;

[11] Tian Leng, He Shunli. Gas water layer identification technology based on improved artificial neural network [J].logging technology, 2009, 33(5): 449-452; 
[12] ZhongDakang, Zhang Guoxi. Application of artificial neural network in identification of mud logging reservoir [J]. Journal of Southwest Petroleum Institute, 2002, 24(3): 28 30

[13] Wang Jinfeng, Yang Chen, Mao Dengtian, Gou Feifei.Prediction of equivalent static density of water-based fuzzy ball drilling fluid by BP neural network method[J].OIL DRILLING \& PRODUCTION TECHNOLOGY, 2013,35(6): 32-35

[14] Wang Lu, Wu Guoping. Oil- Gas Recognition Based on Genetic Algorithm and BP Neural Network [J].CHINESE JOURNAL OF ENGINEERING GEOPHYSICS, 2008, 5(2):169-172

[15] Zhang Tao. Application of BP Neural Network in Logging Interpretation [D].Xi'an: Northwest University, 2010: 1-15

[16] Shi Guangren, Zhang Guangya, Shi Xiaofei. Multi-target optimization of exploration prospects-Application of artificial neural network and multiple regression analysis[D].ACTA PET ROLEI SINICA, 2002,23)(5):19-22 